\title{
Support theorem for the solution of a white-noise-driven parabolic stochastic partial differential equation with temporal Poissonian jumps
}

\author{
NICOLAS FOURNIER \\ Institut Elie Cartan, Université Henri Poincaré, B.P. 239, F-54506 Vandoeuvre-lès-Nancy Cedex. \\ E-mail: fournier@iecn.el-nancy.fr
}

We study the weak solution $X$ of a parabolic stochastic partial differential equation driven by two independent processes: a Gaussian white noise and a finite Poisson measure. We characterize the support of the law of $X$ as the closure in $\mathbb{D}([0, T], C([0,1]))$, endowed with its Skorokhod topology, of a set of weak solutions of ordinary partial differential equations.

Keywords: parabolic stochastic partial differential equations; Poisson measure; support theorem; white noise

\section{Introduction}

Consider a space-time white noise $W(\mathrm{~d} x, \mathrm{~d} t)$ on $[0, T] \times[0,1]$, based on $\mathrm{d} x \mathrm{~d} t$ (see Walsh 1986 , p. 269). Denote by $(E, d)$ a Polish space endowed with a positive finite measure $q$, and by $N(\mathrm{~d} t, \mathrm{~d} z)$ a Poisson measure on $[0, T] \times E$, with intensity measure $\mathrm{d} t q(\mathrm{~d} z)$, independent of $W$. Our purpose is to study the following stochastic partial differential equation $(\mathrm{SPDE})$ on $[0, T] \times[0,1]$ :

$$
\frac{\partial X}{\partial t}(t, x)=\frac{\partial^{2} X}{\partial x^{2}}(t, x)+b(X(t, x))+\sigma(X(t, x)) \dot{W}_{x, t}+\int_{E} g(X(t-, x), z) \dot{N}_{t}(\mathrm{~d} z)
$$

with Neumann boundary conditions

$$
\frac{\partial X}{\partial x}(t, 0)=\frac{\partial X}{\partial x}(t, 1)=0, \quad \forall t>0,
$$

and deterministic initial condition $\mathscr{C}_{0}(x) \in C([0,1])$. The symbols $\dot{N}_{t}(\mathrm{~d} z)$ and $\dot{W}_{t, x}$ stand respectively for the heuristical Radon-Nikodym density of $N(\mathrm{~d} t, \mathrm{~d} z)$ and $W(\mathrm{~d} x, \mathrm{~d} t)$ with respect to the Lebesgue measures $\mathrm{d} t$ and $\mathrm{d} t \mathrm{~d} x$. We could also write, with an abuse of notation, $\dot{N}_{t}(\mathrm{~d} z) \mathrm{d} t \mathrm{~d} x=\mathrm{d} x N(\mathrm{~d} t, \mathrm{~d} z)$ and $\dot{W}_{x, t} \mathrm{~d} t \mathrm{~d} x=W(\mathrm{~d} x, \mathrm{~d} t)$.

We denote by $\mathbb{D}([0, T], C([0,1]))$ the set of cadlag functions from $[0, T]$ into $C([0,1])$, endowed with the corresponding Skorokhod topology. In this paper, we characterize the 
support of the law of a weak solution $X$ of equation (1.1) as the closure of a set of weak solutions of ordinary partial differential equations in $\mathbb{D}([0, T], C([0,1]))$.

Parabolic SPDEs driven by a white noise, i.e. equation (1.1) with $g \equiv 0$, were introduced by Walsh $(1981 ; 1986)$. Walsh (1986) defines his weak solutions, then proves a theorem of existence, uniqueness and regularity. Various properties of Walsh's equation have since been investigated: for example, Malliavin calculus, large deviations and the support theorem (see Bally et al. 1995).

Walsh (1981), however, builds his equation in order to model a discontinuous neurophysiological phenomenon. He explains that the white noise $W$ approximates a Poisson point process. This approximation is realistic because there are many jumps, and the jumps are very small, but in any case the observed phenomenon is discontinuous. However, SPDEs with jumps are much less well known. In the case of temporal and spatial jumps, Saint Loubert Bié (1998) has studied the existence, uniqueness, regularity, and variational calculus. See also Fournier (2000) for other results on the same subject. Nevertheless, no result about the 'joint' regularity of the weak solutions has been proved in this case: we do not really know in which space the weak solution 'lives', thus no support theorem may hold for the moment.

In the case of equation (1.1) with $\sigma \equiv 0$, but with $q(E)=\infty$, and with a compensated Poisson measure, Albeverio et al. (1998) have checked the existence and uniqueness of a 'modified cadlag' weak solution $u(t, x): u$ is almost surely continuous in $x$; and $u$ is rightcontinuous and has left limits in $L^{2}(\Omega)$ in the variable $t$. Again, we do not know in which space the weak solution almost surely lies.

Since Stroock and Varadhan (1972) established their famous support theorem for diffusion processes, there have been many investigations on the subject. In particular, Millet and Sanz-Solé (1994) have considerably simplified the proof of Stroock and Varadhan. But the only support theorem for jump processes seems to be that of Simon (1999), who studies a stochastic differential equation driven by a (compensated or not) infinite Poisson measure. Finally, let us remark that, as far as we know, no support theorem seems to be known in the case of equations driven by two independent (but different) random elements.

This paper is organized as follows. In Section 2, we define the weak solutions of (1.1), following Walsh (1986). Using the method of Ikeda and Watanabe (1979), and applying Walsh's results, we sketch the proof of an existence and uniqueness result. We define the 'skeleton' associated with (1.1), by using the Cameron-Martin space associated with $W$ and the set of finite counting measures associated with $N$. Finally, we state our support theorem.

Section 3 is devoted to a simplification of the problem. First, we use a localization argument in order to obtain weaker assumptions. Then we prove that it suffices to check two simpler support theorems. The first is proved in Section 4, and is related to an equation similar to (1.1) but without white noise: $\dot{W}_{t, x}$ is replaced by $\dot{h}(t, x)$, where $h$ is an element of the Cameron-Martin space associated with $W$. The second is proved in Section 5, and deals with an equation without Poisson measure, but with an additional 'jump drift'. This concludes the proof of our main result.

Section 6 is devoted to an extension of our result to the case where the Poisson measure is almost surely infinite $(q(E)=\infty)$, but where the diffusion coefficient is constant $(\sigma(x)=\sigma)$.

Finally, some technical results are given in the Appendix. 


\section{Framework}

Let us first define the weak solutions of (1.1). To this end, we need some assumptions:

Assumption (H). The functions $\sigma$ and $b: \mathbb{R} \mapsto \mathbb{R}$, satisfy a global Lipschitz condition. The function $g: \mathbb{R} \times E \mapsto \mathbb{R}$ is measurable on $\mathbb{R} \times E$ and, for each $z \in E$, the map $g(., z)$ is continuous on $\mathbb{R}$.

We proceed by following Walsh (1986, pp. 311-322). Consider the Green kernel $G_{t}(x, y)$ associated with the deterministic system

$$
\frac{\partial u}{\partial t}=\frac{\partial^{2} u}{\partial x^{2}}, \quad \frac{\partial u}{\partial x}(t, 0)=\frac{\partial u}{\partial x}(t, 1)=0 .
$$

This kernel can be explicitely computed:

$$
G_{t}(x, y)=\frac{1}{\sqrt{4 \pi t}} \sum_{n \in Z}\left[\exp \left(\frac{-(y-x-2 n L)^{2}}{4 t}\right)+\exp \left(\frac{-(y+x-2 n L)^{2}}{4 t}\right)\right] .
$$

If $\phi$ belongs to $C([0,1])$, we set

$$
G_{t}(\phi, x)=\left\{\begin{array}{cc}
\phi(x) & \text { if } t=0 \\
\int_{0}^{1} G_{t}(x, y) \phi(y) \mathrm{d} y & \text { if } t>0
\end{array}\right.
$$

The Appendix contains technical results about this kernel. We endow our probability space $(\Omega, \mathscr{F}, P)$ with the canonical filtration associated with the independent random elements $W$ and $N$ :

$$
\mathscr{F}_{t}=\sigma\{W(A) ; A \in \mathscr{B}([0,1] \times[0, t])\} \vee \sigma\{N(B) ; B \in \mathscr{B}([0, t] \times E)\} .
$$

A process $X(t, x)$ on $[0, T] \times[0,1]$ is said to be adapted if, for all $t \geqslant 0$, all $x \in[0,1]$, $X(t, x)$ is $\mathscr{F}_{t}$-measurable.

Like Walsh (see also Saint Loubert Bié 1998, or Fournier 2000), we define the weak solutions of (1.1) in the following sense.

Definition 2.1. Let $\mathscr{B}_{0}:[0,1] \mapsto \mathbb{R}$ be a continuous deterministic function. Consider an adapted process $X(t, x)$ on $[0, T] \times[0,1]$, lying almost surely in $\mathbb{D}([0, T], C([0,1]))$. Then $X$ is said to be a weak solution of (1.1) if and only if it satisfies the following evolution equation:

$$
\begin{aligned}
X(t, x)= & G_{t}\left(\mathscr{C}_{0}, x\right)+\int_{0}^{t} \int_{0}^{1} G_{t-s}(x, y)[b(X(s, y)) \mathrm{d} y \mathrm{~d} s+\sigma(X(s, y)) W(\mathrm{~d} y, \mathrm{~d} s)] \\
& +\int_{0}^{t} \int_{E} \int_{0}^{1} G_{t-s}(x, y) g(X(s-, y), z) \mathrm{d} y N(\mathrm{~d} s, \mathrm{~d} z),
\end{aligned}
$$

where $G_{t}\left(\mathscr{C}_{0}, x\right)$ is defined by (2.3), and with the convention that 


$$
\int_{0}^{1} G_{0}(x, y) g(X(s-, y), z) \mathrm{d} y=g(X(s-, x), z) .
$$

We now establish an existence and uniqueness result for such a solution. Since $q(E)$ is finite, $N([0, T] \times E)$ is almost surely finite, and thus $N$ can almost surely be written as

$$
N(\mathrm{~d} t, \mathrm{~d} z)=\sum_{i=1}^{\mu} \delta_{\left(T_{i}, Z_{i}\right)}(\mathrm{d} t, \mathrm{~d} z),
$$

with $\mu \in \mathbb{N}, 0<T_{1}<\ldots<T_{\mu}<T$, and $Z_{1}, \ldots, Z_{\mu} \in E$. Hence, (2.5) can be written as

$$
\begin{aligned}
X(t, x)= & G_{t}\left(\mathscr{C}_{0}, x\right)+\int_{0}^{t} \int_{0}^{1} G_{t-s}(x, y)[b(X(s, y)) \mathrm{d} y \mathrm{~d} s+\sigma(X(s, y)) W(\mathrm{~d} y, \mathrm{~d} s)] \\
& +\sum_{i=1}^{\mu} \mathbf{1}_{\left\{t \geqslant T_{i}\right\}} \int_{0}^{1} G_{t-T_{i}}(x, y) g\left(X\left(T_{i}-, y\right), Z_{i}\right) \mathrm{d} y .
\end{aligned}
$$

Working recursively on the time intervals $\left[0, T_{1}\left[,\left[T_{1}, T_{2}\left[, \ldots,\left[T_{\mu}, T\right]\right.\right.\right.\right.$, as in the proof of Theorem 9.1 in Ikeda and Watanabe (1979, pp. 231-232), using Walsh's theorems of existence, uniqueness and regularity for (1.1) with $g \equiv 0$ (see Theorem 3.2 and Corollary 3.4 in Walsh 1986, pp. 313 and 317), and using the well-known estimates of the Green kernel stated in the Appendix, one can prove the following proposition:

Proposition 2.2. Assume $(H)$. Equation (1.1) admits a unique adapted solution $X(t, x)$ on $[0, T] \times[0,1]$, lying almost surely in $\mathbb{D}([0, T], C([0,1]))$. Uniqueness holds in the sense that if $Y$ is another adapted solution lying in $\mathbb{D}([0, T], C([0,1]))$, then almost surely,

$$
\sup _{[0, T] \times[0,1]}|X(t, x)-Y(t, x)|=0 .
$$

We are now interested in the support of the law of $X$. Let us first recall the definition of the Skorokhod distance on $\mathbb{D}([0, T], C([0,1]))$. We consider the set of 'changes of time':

$$
\Lambda=\{\lambda \in C([0, T]) \mid \lambda(0)=0, \lambda(T)=T, \lambda \text { is strictly increasing }\} .
$$

For $\lambda \in \Lambda$, we set

$$
\||\lambda|\|=\sup _{0 \leqslant s<t \leqslant T}\left|\ln \left\{\frac{\lambda(t)-\lambda(s)}{t-s}\right\}\right| .
$$

The Skorokhod distance between two elements $\phi$ and $\psi$ of $\mathbb{D}([0, T], C([0,1]))$ is given by

$$
\delta(\phi, \psi)=\inf _{\lambda \in \Lambda}\left\{\sup _{[0, T] \times[0,1]}|\phi(\lambda(t), x)-\psi(t, x)|+|||\lambda| \| \mid\right\} .
$$

$\mathbb{D}([0, T], C([0,1]))$, endowed with $\delta$, is a Polish space (see, for example, Jacod and Shiryaev 1987 , p. 289).

We now introduce some notation, describing the 'supports' of $W$ and $N$ : we denote by 


$$
\mathscr{H}=\left\{h(t, x)=\int_{0}^{t} \int_{0}^{x} \dot{h}(s, y) \mathrm{d} y \mathrm{~d} s \mid \dot{h} \in L^{2}([0, T] \times[0,1])\right\}
$$

the Cameron-Martin space associated with $W$. We also consider the set of the finite counting measures on $[0, T] \times E$, the support of which is contained in $[0, T] \times \operatorname{supp} q$ :

$$
\mathscr{M}=\left\{m(\mathrm{~d} t, \mathrm{~d} z)=\sum_{i=1}^{n} \delta_{\left(t_{i}, z_{i}\right)}(\mathrm{d} t, \mathrm{~d} z) \mid n \in \mathbb{N}, 0<t_{1}<\ldots<t_{n}<T, z_{1}, \ldots, z_{n} \in \operatorname{supp} q\right\},
$$

with the convention $\sum_{i=1}^{0}=0$. Notice that, for all $\omega \in \Omega, N(\omega)$ belongs to $\mathscr{M}$. But in general (with an abuse of notation), $\dot{W}(\omega) \notin \mathscr{H}$, since $\dot{W}(\omega)$ is not even well defined.

The following proposition describes the 'skeleton' associated with our evolution equation.

Proposition 2.3. Assume $(H)$. Let $h \in \mathscr{H}$ and $m \in \mathscr{L l}$ be fixed. The following ordinary evolution equation admits a unique solution, which we denote by $S(h, m)$, lying in $\mathbb{D}([0, T], C([0,1]))$ :

$$
\begin{aligned}
S(h, m)(t, x)= & G_{t}\left(\mathscr{C}_{0}, x\right)+\int_{0}^{t} \int_{0}^{1} G_{t-s}(x, y) \\
& \times[b(S(h, m)(s, y)) \mathrm{d} y \mathrm{~d} s+\sigma(S(h, m)(s, y)) \dot{h}(s, y) \mathrm{d} y \mathrm{~d} s] \\
& +\int_{0}^{t} \int_{E} \int_{0}^{1} G_{t-s}(x, y) g(S(h, m)(s-, y), z) \mathrm{d} y m(\mathrm{~d} s, \mathrm{~d} z) .
\end{aligned}
$$

This proposition can be proved similarly to Proposition 2.2. Equation (2.6) is the same as (2.4), but we have replaced $W(\mathrm{~d} y, \mathrm{~d} s)$ and $N(\mathrm{~d} s, \mathrm{~d} z)$ by $\dot{h}(s, y) \mathrm{d} y \mathrm{~d} s$ and $m(\mathrm{~d} s, \mathrm{~d} z)$.

Finally, we recall the following standard observation:

Remark 2.4. Let $Z$ be a random variable with values in a Polish space $A$ endowed with a distance $\alpha$. Recall that the support $\operatorname{supp}_{\alpha} P \circ Z^{-1}$ of the law of $Z$ related to the distance $\alpha$ is the smallest closed subset $F$ of $(A, \alpha)$ satisfying $P(Z \in F)=1$. Let $B$ be a subset of $A$, and let $\bar{B}^{\alpha}$ be its closure in $(A, \alpha)$.

(a) If $Z \in \bar{B}^{\alpha}$ almost surely, then

$$
\operatorname{supp}_{\alpha} P \circ Z^{-1} \subset \bar{B}^{\alpha} .
$$

(b) If, for all $b \in B$, all $\varepsilon>0$,

$$
P(\alpha(b, Z)<\varepsilon)>0,
$$

then

$$
\bar{B}^{\alpha} \subset \operatorname{supp}_{\alpha} P \circ Z^{-1} .
$$

In order to establish a support theorem, we need the following assumptions. 
Assumption (S1). The function $\sigma$ is $C^{3}$ on $\mathbb{R}$.

Assumption (S2). For each $z_{0} \in E$, each $n \in \mathbb{N}$,

$$
\sup _{|x| \leqslant n}\left|g(x, z)-g\left(x, z_{0}\right)\right| \underset{d\left(z, z_{0}\right) \rightarrow 0}{\longrightarrow} 0 .
$$

For each $z_{0} \in E$, each $n \in \mathbb{N}$, there exist a constant $\xi^{n}\left(z_{0}\right)>0$ and a function $\psi_{z_{0}}^{n}(u)$ : $\mathbb{R}^{+} \mapsto \mathbb{R}^{+}$, decreasing to 0 when $u$ decreases to 0 , such that, for all $|x| \leqslant n,|y| \leqslant n$,

$$
\sup _{d\left(z, z_{0}\right) \leqslant \xi^{n}\left(z_{0}\right)}|g(x, z)-g(y, z)| \leqslant \psi_{z_{0}}^{n}(|x-y|) .
$$

Assumption $(S 1)$ is nearly the same as that of Bally et al. (1995), who prove a support theorem in the case where $g \equiv 0$, and comes from a Taylor expansion of order 3. In fact they assume that $\sigma$ is $C_{b}^{3}$, but a localization procedure can be done (see the proof of Proposition 3.1 in the next section). Assumption ( $S 2)$ says that $g$ is locally uniformly continuous. In the particular case where $E$ is locally compact, $(S 2)$ is satisfied as soon as $g$ is continuous on $[0, T] \times E$.

Now we can state our main result:

Theorem 2.5. Under $(H),(S 1)$ and $(S 2)$, if $X$ denotes the unique weak solution of equation (1.1),

$$
\operatorname{supp}_{\delta} P \circ X^{-1}=\overline{\{S(h, m) \mid h \in \mathscr{H}, m \in \mathscr{C}\}^{\delta}} .
$$

\section{Simplification of the problem}

First, we 'delocalize' Assumptions $(S 1)$ and $(S 2)$, by using a standard argument. Consider the following assumptions, stronger than $(S 1)$ and $(S 2)$.

Assumption ( $\left.\boldsymbol{S}^{\prime} 1\right)$. The function $\sigma$ is $C^{3}$ on $\mathbb{R}$, bounded with its derivatives.

Assumption ( $\left.\boldsymbol{S}^{\prime} 2\right)$. For all $z_{0} \in E$,

$$
\sup _{x \in \mathbb{R}}\left|g\left(x, z_{0}\right)\right|<\infty, \quad \sup _{x \in \mathbb{R}}\left|g(x, z)-g\left(x, z_{0}\right)\right| \underset{d\left(z, z_{0}\right) \rightarrow 0}{\rightarrow} 0 .
$$

For all $z_{0} \in E$, there exist $\xi\left(z_{0}\right)>0$ and a function $\psi_{z_{0}}(u): \mathbb{R}^{+} \mapsto \mathbb{R}^{+}$, decreasing to 0 when $u$ decreases to 0 , such that, for all $x, y \in \mathbb{R}$,

$$
\sup _{d\left(z, z_{0}\right) \leqslant \xi\left(z_{0}\right)}|g(x, z)-g(y, z)| \leqslant \psi_{z_{0}}(|x-y|) .
$$

Proposition 3.1. If Theorem 2.5 holds under $(H),\left(S^{\prime} 1\right)$ and $\left(S^{\prime} 2\right)$, then it also holds under $(H),(S 1)$ and $(S 2)$.

We will prove this proposition at the end of the section. 
We would now like to check that Theorem 2.5 holds as soon as two easier support theorems are valid. The first deals with equation (2.4) with a 'deterministic' white noise, and the second with a 'deterministic' Poisson measure.

We first introduce some notation. If respectively $h \in \mathscr{H}$ and $m \in \mathscr{C l}$, then we denote by $X_{h}$ and $X_{m}$ the solution of (2.4), where we have replaced $W(\mathrm{~d} y, \mathrm{~d} s)$ by $\dot{h}(s, y) \mathrm{d} y \mathrm{~d} s$ and $N(\mathrm{~d} t, \mathrm{~d} z)$ by $m(\mathrm{~d} t, \mathrm{~d} z)$. In other words,

$$
\begin{aligned}
X_{h}(t, x)= & G_{t}\left(\mathscr{C}_{0}, x\right)+\int_{0}^{t} \int_{0}^{1} G_{t-s}(x, y)\left[b\left(X_{h}(s, y)\right) \mathrm{d} y \mathrm{~d} s+\sigma\left(X_{h}(s, y)\right) \dot{h}(s, y) \mathrm{d} y \mathrm{~d} s\right] \\
& +\int_{0}^{t} \int_{E} \int_{0}^{1} G_{t-s}(x, y) g\left(X_{h}(s-, y), z\right) \mathrm{d} y N(\mathrm{~d} s, \mathrm{~d} z), \\
X_{m}(t, x)= & G_{t}\left(\mathscr{C}_{0}, x\right)+\int_{0}^{t} \int_{0}^{1} G_{t-s}(x, y)\left[b\left(X_{m}(s, y)\right) \mathrm{d} y \mathrm{~d} s+\sigma\left(X_{m}(s, y)\right) W(\mathrm{~d} y, \mathrm{~d} s)\right] \\
& +\int_{0}^{t} \int_{E} \int_{0}^{1} G_{t-s}(x, y) g\left(X_{m}(s-, y), z\right) \mathrm{d} y m(\mathrm{~d} s, \mathrm{~d} z) .
\end{aligned}
$$

We could also write, with an abuse of notation, $X_{h}=S(h, N)$ and $X_{m}=S(\dot{W}, m)$. The next sections are devoted to the proof of the following propositions.

Proposition 3.2. Assume $(H)$ and $\left(S^{\prime} 2\right)$. Let $h \in \mathscr{H}, m \in \mathscr{l}$, and $\epsilon>0$ be fixed. Then

$$
P\left(\delta\left(S(h, m), X_{h}\right) \leqslant \varepsilon\right)>0 .
$$

We now denote by $\|u\|_{\infty}=\sup _{[0, T] \times[0,1]}|u(t, x)|$ the supremum norm on $[0, T] \times[0,1]$.

Proposition 3.3. Assume $(H),\left(S^{\prime} 1\right)$ and $\left(S^{\prime} 2\right)$. Let $m \in \mathscr{l l}$ be fixed. Then

$$
\operatorname{supp}_{\|\|_{\infty}} P \circ X_{m}^{-1}=\overline{\{S(h, m) \mid h \in \mathscr{H}\}^{\|} \|_{\infty}} .
$$

Let us observe that this second result implies the following weaker one:

$$
\operatorname{supp}_{\delta} P \circ X_{m}^{-1}=\overline{\{S(h, m) \mid h \in \mathscr{H}\}} \delta
$$

Assuming for a moment that these propositions hold, we prove our main result.

Proof of Theorem 2.5. Using Remark 2.4, we tackle the proof in two stages.

We first check that $X$ almost surely belongs to $\overline{\{S(h, m) \mid h \in \mathscr{H}, m \in \mathscr{C}\}} \delta$. Consider the map from $\mathscr{C}$ to $[0,1]$ defined by

$$
\phi(\mu)=P\left(X_{\mu} \in \overline{\{S(h, m) \mid h \in \mathscr{H}, m \in \mathscr{C}\}^{\delta}}\right) .
$$

Let us first prove that

$$
P(X \in \overline{\{S(h, m) \mid h \in \mathscr{H}, m \in \mathscr{C l}\}} \delta \mid \sigma(N))=\phi(N)
$$


almost surely, where

$$
\sigma(N)=\sigma\{N(A) ; A \in \mathscr{B}([0, T] \times E)\} .
$$

In order to understand (3.4), let us work with the canonical product space

$$
(\Omega, \mathscr{F}, P)=\left(\Omega^{W}, \mathscr{F}^{W}, P^{W}\right) \otimes\left(\Omega^{N}, \mathscr{F}^{N}, P^{N}\right)
$$

associated with $W$ and $N$. Every element $\omega$ of $\Omega$ can be written as $\left(\omega^{W}, \omega^{N}\right)$, where $\omega^{W} C([0, T] \times[0,1])$ and $\omega^{N} \in \mathscr{M l}$. Thus,

$$
P(X \in \overline{\{S(h, m) \mid h \in \mathscr{H}, m \in \mathscr{C} \zeta\}} \delta \mid \sigma(N))(\omega)=\int \mathbf{1}_{\left\{X\left(\omega^{W}, \omega^{N}\right) \in \overline{\{S(h, m) \mid h \in \mathscr{H}, m \in \mathscr{M}\}} \delta\right\}} \mathrm{d} P^{W}\left(\omega^{W}\right) .
$$

But obviously, $X(\omega)=X\left(\omega^{W}, \omega^{N}\right)=X_{\omega^{N}}\left(\omega^{W}\right)$, where $X_{\mu}$ was defined by (3.3) for each $\mu \in \mathscr{L l}$. Thus,

$$
P\left(X \in \overline{\{S(h, m) \mid h \in \mathscr{H}, m \in \mathscr{C}\}}^{\delta} \mid \sigma(N)\right)(\omega)=P^{W}\left(X_{\omega^{N}} \in \overline{\{S(h, m) \mid h \in \mathscr{H}, m \in \mathscr{C l}\}^{\delta}}\right) .
$$

Now, since for each $\mu \in \mathscr{L}, X_{\mu}$ is independent of $N$, we notice that

$$
\phi(\mu)=P^{W}\left(X_{\mu} \in \overline{\{S(h, m) \mid h \in \mathscr{H}, m \in \mathscr{C}\}}{ }^{\delta}\right) .
$$

Comparing (3.5) and (3.6), we deduce (3.4). Hence, we obtain

$$
P(X \in \overline{\{S(h, m) \mid h \in \mathscr{H}, m \in \mathscr{C l}\}} \delta)=\mathrm{E}(\phi(N)) .
$$

Finally, it is clear from the definition of $\phi$ and from Proposition 3.3 that $\phi \equiv 1$. The conclusion follows easily.

We now fix $h \in \mathscr{H}, m \in \mathscr{C}$ and $\epsilon>0$. We have to check that

$$
P_{0}=P(\delta(X, S(h, m)) \leqslant \varepsilon)>0 .
$$

First,

$$
P_{0} \geqslant P\left(\delta\left(X, X_{h}\right) \leqslant \varepsilon / 2 ; \delta\left(X_{h}, S(h, m)\right) \leqslant \varepsilon / 2\right) .
$$

Noticing that $X_{h}$ is $\sigma(N)$-measurable, we see that

$$
P_{0} \geqslant \mathrm{E}\left[\mathbf{1}_{\left\{\delta\left(X_{\mathrm{h}}, S(h, m)\right) \leqslant \varepsilon / 2\right\}} P\left(\delta\left(X, X_{h}\right) \leqslant \varepsilon / 2 \mid \sigma(N)\right)\right] .
$$

But we know from Proposition 3.3 that, for all $m \in \mathscr{L}$,

$$
\psi(m)=P\left(\delta\left(X_{m}, S(h, m)\right) \leqslant \varepsilon / 2\right)>0 .
$$

Working on the canonical product space as in the previous paragraph, and noticing that, for all $\omega=\left(\omega^{W}, \omega^{N}\right) \in \Omega, X(\omega)=X_{\omega^{N}}\left(\omega^{W}\right)$ and $X_{h}(\omega)=S\left(h, \omega^{N}\right)-$ all of this with no abuse of notation - we deduce that

$$
P\left(\delta\left(X, X_{h}\right) \leqslant \varepsilon / 2 \mid \sigma(N)\right)=\psi(N)>0
$$

almost surely. Thus, (3.7) holds as soon as

$$
P\left(\delta\left(X_{h}, S(h, m)\right) \leqslant \varepsilon / 2\right)>0,
$$


which never fails, thanks to Proposition 3.2.

Provided we check Propositions 3.1, 3.2 and 3.3, Theorem 2.5 is proved.

In order to prove Proposition 3.1, we begin with a lemma.

Lemma 3.4. Consider some functions $\sigma, b$ and $g(\bar{\sigma}, \bar{b}$ and $\bar{g})$ satisfying Assumption $(H)$, and denote by $X(\bar{X})$ the corresponding unique weak solution of (1.1). Assume that, for some $A \in \mathbb{R}^{+}$,

$$
\forall|x| \leqslant A, \quad \forall z \in E, \quad \sigma(x)=\bar{\sigma}(x), \quad b(x)=\bar{b}(x), \quad g(x, z)=\bar{g}(x, z) .
$$

Then there exists $\tilde{\Omega} \subset \Omega$ such that $P(\tilde{\Omega})=1$ and

$$
\left\{\tilde{\omega} \in \Omega ;\|X(\omega)\|_{\infty} \leqslant A\right\} \subset\left\{\omega \in \tilde{\Omega} ;\|X(\omega)-\bar{X}(\omega)\|_{\infty}=0\right\} .
$$

Proof. We consider the stopping time $\tau=\inf \left\{t \geqslant 0, \sup _{x}|X(t, x)| \geqslant A\right\}$. Then the processes $X^{\tau}(t, x)=X(t \wedge \tau, x)$ and $\bar{X}^{\tau}(t, x)=\bar{X}(t \wedge \tau, x)$ satisfy the same evolution equation:

$$
\begin{aligned}
X^{\tau}(t, x)= & G_{t}\left(\mathscr{C}_{0}, x\right)+\int_{0}^{t \wedge \tau} \int_{0}^{1} G_{t-s}(x, y)\left[\bar{b}\left(X^{\tau}(s, y)\right) \mathrm{d} y \mathrm{~d} s+\bar{\sigma}\left(X^{\tau}(s, y)\right) W(\mathrm{~d} y, \mathrm{~d} s)\right] \\
& +\int_{0}^{t \wedge \tau} \int_{E} \int_{0}^{1} G_{t-s}(x, y) \bar{g}\left(X^{\tau}(s-, y), z\right) \mathrm{d} y N(\mathrm{~d} s, \mathrm{~d} z) .
\end{aligned}
$$

A uniqueness argument yields that almost surely, say for all $\omega \in \tilde{\Omega}$, with $P(\tilde{\Omega})=1, X^{\tau}=\bar{X}^{\tau}$ on $[0, T] \times[0,1]$. This yields that, for all $\omega \in \tilde{\Omega}$, all $t \leqslant \tau$, and all $x \in[0,1], X(t, x)=$ $\bar{X}(t, x)$. This implies that

$$
\left\{\omega \in \tilde{\Omega} ;\|X(\omega)\|_{\infty} \leqslant A\right\} \subset\{\omega \in \tilde{\Omega} ; \tau(\omega)>T\} \subset\left\{\omega \in \tilde{\Omega} ;\|X(\omega)-\bar{X}(\omega)\|_{\infty}=0\right\} .
$$

Proof of Proposition 3.1. We assume that Theorem 2.5 holds under $(H),\left(S^{\prime} 1\right)$ and $\left(S^{\prime} 2\right)$, and we consider functions $b, \sigma$ and $g$ satisfying only $(H),(S 1)$ and $(S 2)$. We need a sequence of $C_{b}^{\infty}$ functions $\phi_{n}: \mathbb{R} \mapsto[0,1]$, satisfying

$$
\phi_{n}(x)= \begin{cases}1 & \text { if }|x| \leqslant n \\ 0 & \text { if }|x| \geqslant n+1\end{cases}
$$

Then the functions $\sigma_{n}(x)=\sigma(x) \phi_{n}(x)$ and $g_{n}(x, z)=g(x, z) \phi_{n}(x)$ clearly satisfy $\left(S^{\prime} 1\right)$ and $\left(S^{\prime} 2\right)$. Denote by $X_{n}$ the solution of (2.4) with $\sigma_{n}$ and $g_{n}$ instead of $\sigma$ and $g$. Lemma 3.4 yields that there exists $\tilde{\Omega} \subset \Omega$ such that $P(\tilde{\Omega})=1$ and, for all $n \in \mathbb{N}$,

$$
\left\{\omega \tilde{\Omega} ;\|X(\omega)\|_{\infty} \leqslant n\right\} \subset\left\{\omega \in \tilde{\Omega} ;\left\|X(\omega)-X_{n}(\omega)\right\|_{\infty}=0\right\}
$$

In the same way, we define $S_{n}(h, m)$, for $h \in \mathscr{H}$ and $m \in \mathscr{L}$, as the solution of equation (2.6) with $\sigma_{n}$ and $g_{n}$ instead of $\sigma$ and $g$. We obtain, for all $n \in \mathbb{N}$,

$$
\text { if }\|S(h, m)\|_{\infty} \leqslant n \text { or }\left\|S_{n}(h, m)\right\|_{\infty} \leqslant n \text {, then } S(h, m)=S_{n}(h, m) \text {. }
$$

Since Theorem 2.5 holds under $(H),\left(S^{\prime} 1\right)$, and $\left(S^{\prime} 2\right)$, we know that for each $n \in \mathbb{N}$, 


$$
\operatorname{supp}_{\delta} P \circ X_{n}^{-1}=\overline{\left\{S_{n}(h, m) \mid h \in \mathscr{H}, m \in \mathscr{C}\right\}^{\delta}} .
$$

Using Remark 2.4, Proposition 3.1 will hold if we check, on the one hand, that

$$
\left.P(X \in \overline{\{S(h, m) \mid h \in \mathscr{H}, m \in \mathscr{C}\}}\}^{\delta}\right)=1
$$

and, on the other hand, that for all $h \in \mathscr{H}$, all $m \in \mathscr{N}$, all $\epsilon>0$,

$$
P(\delta(X, S(h, m)) \leqslant \epsilon)>0 .
$$

Let us first prove (3.10). Let $\omega \in \tilde{\Omega}$ be fixed. Since $X(\omega)$ belongs to $\mathbb{D}([0, T], C([0,1]))$, it is bounded, and there exists $n \in \mathbb{N}$ (depending on $\omega$ ) such that

$$
n \geqslant\|X(\omega)\|_{\infty}+1
$$

which yields $X(\omega)=X_{n}(\omega)$. But for all $\epsilon>0$, we know from (3.9) that, for almost all $\omega \in \tilde{\Omega}$, there exists $h \in \mathscr{H}$ and $m \in \mathscr{C}$ (depending on $\omega$ ) such that

$$
\delta\left(X_{n}(\omega), S_{n}(h, m)\right) \leqslant \varepsilon .
$$

This and (3.12) yield (if $\varepsilon \leqslant 1$ ) that $\left\|S_{n}(h, m)\right\|_{\infty} \leqslant n$, and thus that $S_{n}(h, m)=S(h, m)$. Hence

$$
\delta(X(\omega), S(h, m)) \leqslant \varepsilon,
$$

which concludes the proof of (3.30), since $P(\tilde{\Omega})=1$.

In order to prove (3.31), we fix $h \in \mathscr{H}, m \in \mathscr{C}$ and $\epsilon>0$. We consider $n \in \mathbb{N}$ such that

$$
n \geqslant\|S(h, m)\|_{\infty}+1 \text {. }
$$

Thus, if $\varepsilon<1$,

$$
\begin{aligned}
P(\delta(X, S(h, m)) \leqslant \epsilon) & =P\left(\delta\left(X, S_{n}(h, m)\right) \leqslant \varepsilon\right) \\
& =P\left(\|X\|_{\infty} \leqslant n, \delta\left(X, S_{n}(h, m)\right) \leqslant \varepsilon\right) \\
& =P\left(\delta\left(X_{n}, S_{n}(h, m)\right) \leqslant \varepsilon\right)
\end{aligned}
$$

thanks to (3.8). From (3.9), this probability is strictly positive, which yields (3.11). Proposition 3.1 is proved.

\section{The case where $W$ is 'deterministic'}

This section is devoted to the proof of Proposition 3.2. We partially follow here the method of Simon (1999), who studies the support of Poisson-driven SDEs (without Wiener term). The extension of his method to SPDEs leads to technical problems, essentially because we have to control the explosion of the Green kernel $G_{t}(x, y)$. Another new difficulty arises because we have to add a second drift, in which the term $\dot{h}(s, y)$ belongs only to $L^{2}([0, T] \times[0,1])$.

In this entire section, 


$$
h(t, x)=\int_{0}^{t} \int_{0}^{x} \dot{h}(s, y) \mathrm{d} y \mathrm{~d} s \in \mathscr{H} \quad \text { and } \quad m(\mathrm{~d} t, \mathrm{~d} z)=\sum_{i=1}^{n} \delta_{\left(t_{i}, z_{i}\right)}(\mathrm{d} t, \mathrm{~d} z) \in \mathscr{C}
$$

are fixed. We set $t_{0}=0, t_{n+1}=T$ and

$$
\zeta_{0}=\inf _{i=0, \ldots, n}\left|t_{i+1}-t_{i}\right|>0 .
$$

For simplicity, we set $S=S(h, m)$. We denote by $0<T_{1}(\omega)<\ldots<T_{\mu(\omega)}(\omega)$ the successive jump times of $N(\omega)$, and by $Z_{1}(\omega), \ldots, Z_{\mu(\omega)}(\omega)$ the jump sizes. In other words,

$$
N(\omega, \mathrm{d} t, \mathrm{~d} z)=\sum_{i=1}^{\mu(\omega)} \delta_{\left(T_{i}(\omega), Z_{i}(\omega)\right)}(\mathrm{d} t, \mathrm{~d} z) .
$$

We recall that, for all $\alpha \in] 0, \zeta_{0}[$ and all $\xi>0$, the set

$$
\Omega(\alpha, \xi)=\left\{\omega \in \Omega \mid \mu(\omega)=n, t_{i}-\alpha<T_{i}(\omega)<t_{i}, d\left(z_{i}, Z_{i}(\omega)\right) \leqslant \xi\right\}
$$

has a strictly positive probability. We will check that, for all $\varepsilon>0$, there exist $\alpha>0$ and $\xi>0$ such that, for all $\omega \in \Omega(\alpha, \xi)$,

$$
\delta\left(X_{h}(\omega), S\right) \leqslant \varepsilon
$$

which will imply Proposition 3.2.

Also in this entire section, the constant $C$ depends only on $h, m$ and the parameters $(\sigma$, $b, g, \mathscr{B}_{0}$ and $T$ ) of equation (1.1).

From now on, we consider $\omega \in \Omega(\alpha, \xi)$.

First, we choose $0<\alpha<\xi_{0} / 16$, and $0<\xi<\xi\left(z_{1}\right) \wedge \ldots \wedge \xi\left(z_{n}\right)$, where $\xi\left(z_{i}\right)$ was defined in Assumption $\left(S^{\prime} 2\right)$. For some $\left.\gamma \in\right] 2 \alpha, \zeta_{0} / 8$ [, to be chosen later, we define the polygonal change in time $\lambda \in \Lambda$ by $\lambda(0)=0, \lambda(T)=T$, and, for all $i \in\{1, \ldots, n\}$,

$$
\lambda\left(T_{i}-\gamma\right)=T_{i}-\gamma, \quad \lambda\left(T_{i}\right)=t_{i}, \quad \lambda\left(T_{i}+\gamma\right)=t_{i}+\gamma, \quad \lambda\left(T_{i}+2 \gamma\right)=T_{i}+2 \gamma .
$$

Notice that all the following properties hold:

$$
\begin{aligned}
& \text { for all } t \in\left[T_{i}, T_{i}+\gamma\right], \quad \lambda(t)-t_{i}=t-T_{i} ; \\
& \qquad \begin{array}{l}
\int_{0}^{T} \mathbf{1}_{\{\lambda(s) \neq s\}} \mathrm{d} s \leqslant 3 n \gamma ; \\
\text { for all } t \in[0, T], \quad \lambda(t) \geqslant t \quad \text { and } \quad \mathbf{1}_{\left\{\lambda(t) \geqslant t_{i}\right\}}=\mathbf{1}_{\left\{t \geqslant T_{i}\right\}} ; \\
\|\lambda-I\|_{\infty} \leqslant \alpha .
\end{array}
\end{aligned}
$$

Furthermore, it is easy to check that

$$
|||\lambda||| \leqslant|\ln (1-\alpha / \gamma)| \vee|\ln (1+\alpha / \gamma)| \leqslant 2 \alpha / \gamma,
$$

where the last inequality holds because $\alpha / \gamma \leqslant 1 / 2$. We have to prove that if $\alpha>0$ and $\xi>0$ are small enough then, for some well-chosen $\gamma$,

$$
\left\|S(\lambda(t), x)-X_{h}(t, x)\right\|_{\infty}+\||\lambda|\| \leqslant \varepsilon .
$$


We now set $S_{\lambda}(t, x)=S(\lambda(t), x)$. Then, using (4.3), we see that, for any $\omega \in \Omega(\alpha, \xi)$,

$$
\begin{aligned}
& S_{\lambda}(t, x)-X_{h}(t, x)=G_{\lambda(t)}\left(\mathscr{C}_{0}, x\right)-G_{t}\left(\mathscr{C}_{0}, x\right) \\
& \quad+\int_{0}^{t} \int_{0}^{1}\left(G_{\lambda(t)-s}(x, y)-G_{t-s}(x, y)\right)[b(S(s, y))+\sigma(S(s, y)) \dot{h}(s, y)] \mathrm{d} y \mathrm{~d} s \\
& \quad+\int_{t}^{\lambda(t)} \int_{0}^{1} G_{\lambda(t)-s}(x, y)[b(S(s, y))+\sigma(S(s, y)) \dot{h}(s, y)] \mathrm{d} y \mathrm{~d} s \\
& +\int_{0}^{t} \int_{0}^{1} G_{t-s}(x, y)\left[\left\{b(S(s, y))-b\left(S_{\lambda}(s, y)\right)\right\}+\left\{\sigma(S(s, y))-\sigma\left(S_{\lambda}(s, y)\right)\right\} \dot{h}(s, y)\right] \mathrm{d} y \mathrm{~d} s \\
& \quad+\int_{0}^{t} \int_{0}^{1} G_{t-s}(x, y)\left[\left\{b\left(S_{\lambda}(s, y)\right)-b\left(X_{h}(s, y)\right)\right\}+\left\{\sigma\left(S_{\lambda}(s, y)\right)-\sigma\left(X_{h}(s, y)\right)\right\} \dot{h}(s, y)\right] \mathrm{d} y \mathrm{~d} s \\
& \quad+\sum_{i=1}^{n} \mathbf{1}_{\left\{t \geqslant T_{i}\right\}} \int_{0}^{1}\left(G_{\lambda(t)-t_{i}}(x, y)-G_{t-T_{i}}(x, y)\right) g\left(S\left(t_{i}-, y\right), z_{i}\right) \mathrm{d} y \\
& \quad+\sum_{i=1}^{n} \mathbf{1}_{\left\{t \geqslant T_{i}\right\}} \int_{0}^{1} G_{t-T_{i}}(x, y)\left[g\left(S\left(t_{i}-, y\right), z_{i}\right)-g\left(S\left(t_{i}-, y\right), Z_{i}\right)\right] \mathrm{d} y \\
& \quad+\sum_{i=1}^{n} \mathbf{1}_{\left\{t \geqslant T_{i}\right\}} \int_{0}^{1} G_{t-T_{i}}(x, y)\left[g\left(S\left(t_{i}-, y\right), Z_{i}\right)-g\left(X_{h}\left(T_{i}-, y\right), Z_{i}\right)\right] \mathrm{d} y \\
& =A(t, x)+\ldots+H(t, x) .
\end{aligned}
$$

We compute these terms one by one, still assuming that $\omega \in \Omega(\alpha, \xi)$.

Since $\lambda(t)=t$ for all $t \leqslant T_{1}-\gamma$, and hence for all $t \leqslant 13 \xi_{0} / 16$,

$$
|A(t, x)| \leqslant|A(t, x)| \mathbf{1}_{\left\{t \geqslant 13 \xi_{0} / 16\right\}} \leqslant\left\|\mathscr{C}_{0}\right\|_{\infty} \mathbf{1}_{\left\{t \geqslant 13 \xi_{0} / 16\right\}} \int_{0}^{1}\left|G_{\lambda(t)}(x, y)-G_{t}(x, y)\right| \mathrm{d} y .
$$

Using (A.3) in the Appendix, and then (4.4), we see that

$$
|A(t, x)| \leqslant C \frac{\lambda(t)-t}{\left(13 \xi_{0} / 16\right)^{3 / 2}} \leqslant C\|\lambda-I\|_{\infty} \leqslant C \alpha
$$

Using the Cauchy-Schwarz inequality, then (A.4), and finally (4.4), we obtain 


$$
\begin{aligned}
|B(t, x)| \leqslant & \left(\int_{0}^{t} \int_{0}^{1}[b(S(s, y))+\sigma(S(s, y)) \dot{h}(s, y)]^{2} \mathrm{~d} y \mathrm{~d} s\right)^{1 / 2} \\
& \times\left(\int_{0}^{t} \int_{0}^{1}\left[G_{\lambda(t)-s}(x, y)-G_{t-s}(x, y)\right]^{2} \mathrm{~d} y \mathrm{~d} s\right)^{1 / 2} \\
\leqslant & C(\sqrt{\lambda(t)-t})^{1 / 2} \leqslant C \alpha^{1 / 4}
\end{aligned}
$$

In exactly in the same way, $|C(t, x)| \leqslant C \alpha^{1 / 4}$.

Using Assumption $(H)$, we see that

$$
|D(t, x)| \leqslant C \int_{0}^{t} \int_{0}^{1} G_{t-s}(x, y)\left|S(s, y)-S_{\lambda}(s, y)\right|(1+|\dot{h}(s, y)|) \mathrm{d} y \mathrm{~d} s .
$$

Thanks to the Cauchy-Schwarz inequality and (A.1),

$$
\begin{aligned}
|D(t, x)| & \leqslant C\left(\int_{0}^{t} \sup _{y \in[0,1]}\left|S(s, y)-S_{\lambda}(s, y)\right|^{2} \mathrm{~d} s \int_{0}^{1} G_{t-s}^{2}(x, y) \mathrm{d} y\right)^{1 / 2} \\
& \leqslant C\left(\int_{0}^{t} \mathbf{1}_{\{\lambda(s) \neq s\}} \frac{\mathrm{d} s}{\sqrt{t-s}}\right)^{1 / 2} .
\end{aligned}
$$

Using the Hölder inequality with $p=3$ and $q=3 / 2$, we deduce that

$$
\begin{aligned}
|D(t, x)| & \leqslant C\left(\int_{0}^{t} \mathbf{1}_{\{\lambda(s) \neq s\}} \mathrm{d} s\right)^{1 / 6}\left(\int_{0}^{t} \frac{\mathrm{d} s}{(t-s)^{3 / 4}}\right)^{1 / 3} \\
& \leqslant C\left(\int_{0}^{t} \mathbf{1}_{\{\lambda(s) \neq s\}} \mathrm{d} s\right)^{1 / 6} \leqslant C(3 n \gamma)^{1 / 6} \leqslant C \gamma^{1 / 6}
\end{aligned}
$$

thanks to (4.2). The same computation leads us to

$$
|E(t, x)| \leqslant C\left(\int_{0}^{t} \sup _{y \in[0,1]}\left|S_{\lambda}(s, y)-X_{h}(s, y)\right|^{2} \frac{\mathrm{d} s}{\sqrt{t-s}}\right)^{1 / 2} .
$$

Using (4.1), and (3.1) in $\left(S^{\prime} 2\right)$, we see that

$$
|F(t, x)| \leqslant C \sum_{i=1}^{n} \mathbf{1}_{\left\{t \geqslant T_{i}+\gamma\right\}} \sup _{x, y \in[0,1]}\left|G_{\lambda(t)-t_{i}}(x, y)-G_{t-T_{i}}(x, y)\right| .
$$

Thus, thanks to (A.3),

$$
|F(t, x)| \leqslant C \sum_{i=1}^{n} \mathbf{1}_{\left\{t \geqslant T_{i}+\gamma\right\}} \frac{\left|\left(\lambda(t)-t_{i}\right)-\left(t-T_{i}\right)\right|}{\left[\left(\lambda(t)-t_{i}\right) \wedge\left(t-T_{i}\right)\right]^{3 / 2}} .
$$


But $t \geqslant T_{i}+\gamma$ implies that $\lambda(t)-t_{i} \geqslant \lambda\left(T_{i}+\gamma\right)-t_{i}=\gamma$. Hence, thanks to (4.4) and since $\omega \in \Omega(\alpha, \xi)$,

$$
|F(t, x)| \leqslant C \frac{\|\lambda-I\|_{\infty}+\sup _{i}\left|t_{i}-T_{i}\right|}{\gamma^{3 / 2}} \leqslant C \alpha / \gamma^{3 / 2}
$$

Using (A.2), we deduce that

$$
|G(t, x)| \leqslant \sum_{i=1}^{n} \sup _{y}\left|g\left(S\left(t_{i}-, y\right), z_{i}\right)-g\left(S\left(t_{i}-, y\right), Z_{i}\right)\right| .
$$

Thanks to (3.1) in $\left(S^{\prime} 2\right)$, recalling that for all $i, d\left(z_{i}, Z_{i}\right) \leqslant \xi$, we see that there exists a function $\varphi(\xi)$ from $\mathbb{R}^{+}$into itself, decreasing to 0 when $\xi$ decreases to 0 , depending only on $h, m$ and the parameters of (1.1), such that

$$
|G(t, x)| \leqslant \varphi(\xi)
$$

In the same way, but using (3.2) and the fact that $\xi \leqslant \xi\left(z_{1}\right) \wedge \ldots \wedge \xi\left(z_{n}\right)$, we easily prove the existence of a function $\beta(u): \mathbb{R}^{+} \mapsto \mathbb{R}^{+}$, decreasing to 0 when $u$ decreases to 0 , such that

$$
\begin{aligned}
|H(t, x)| & \leqslant \sum_{i=1}^{n} \mathbf{1}_{\left\{t \geqslant T_{i}\right\}} \times \beta\left(\sup _{y \in[0,1]}\left|S\left(t_{i}-, y\right)-X_{h}\left(T_{i}-, y\right)\right|\right) \\
& \leqslant \sum_{i=1}^{n} \mathbf{1}_{\left\{t \geqslant T_{i}\right\}} \times \beta\left(\sup _{y \in[0,1]}\left|S_{\lambda}\left(T_{i}-, y\right)-X_{h}\left(T_{i}-, y\right)\right|\right)
\end{aligned}
$$

since $\lambda\left(T_{i}\right)=t_{i}$.

Finally, setting

$$
I(t)=\sup _{y \in[0,1]}\left|S_{\lambda}(t, y)-X_{h}(t, y)\right|
$$

and

$$
K(\alpha, \gamma, \xi)=\alpha^{1 / 4} / \gamma^{3 / 2}+\gamma^{1 / 4}+\varphi(\xi),
$$

we obtain

$$
I(t) \leqslant C K(\alpha, \gamma, \xi)+C\left(\int_{0}^{t} I^{2}(s) \frac{\mathrm{d} s}{\sqrt{t-s}}\right)^{1 / 2}+C \sum_{i=1}^{n} \mathbf{1}_{\left\{t \geqslant T_{i}\right\}} \beta\left(I\left(T_{i}-\right)\right) .
$$

Hence

$$
I^{2}(t) \leqslant C K^{2}(\alpha, \gamma, \xi)+C \int_{0}^{t} I^{2}(s) \frac{\mathrm{d} s}{\sqrt{t-s}}+C \sum_{i=1}^{n} \mathbf{1}_{\left\{t \geqslant T_{i}\right\}} \beta^{2}\left(I\left(T_{i}-\right)\right)
$$


Iterating this formula once, we get

$$
\begin{aligned}
I^{2}(t) \leqslant & C K^{2}(\alpha, \gamma, \xi)+C \sum_{i=1}^{n} \mathbf{1}_{\left\{t \geqslant T_{i}\right\}} \beta^{2}\left(I\left(T_{i}-\right)\right) \\
& +C \int_{0}^{t}\left[C K^{2}(\alpha, \gamma, \xi)+C \int_{0}^{s} I^{2}(u) \frac{\mathrm{d} u}{\sqrt{s-u}}+C \sum_{i=1}^{n} \mathbf{1}_{\left\{s \geqslant T_{i}\right\}} \beta^{2}\left(I\left(T_{i}-\right)\right)\right] \frac{\mathrm{d} s}{\sqrt{t-s}} .
\end{aligned}
$$

Using Fubini's theorem, and noticing that $\int_{u}^{t} \mathrm{~d} s / \sqrt{t-s} \sqrt{s-u} \leqslant 4$, we deduce that

$$
I^{2}(t) \leqslant C K^{2}(\alpha, \gamma, \xi)+C \int_{0}^{t} I^{2}(u) \mathrm{d} u+C \sum_{i=1}^{n} \mathbf{1}_{\left\{t \geqslant T_{i}\right\}} \beta^{2}\left(I\left(T_{i}-\right)\right) .
$$

We now apply Gronwall's lemma on $\left[0, T_{1}[\right.$. This gives

$$
\sup _{\left[0, T_{1}[\right.} I^{2}(t) \leqslant C K^{2}(\alpha, \gamma, \xi) \mathrm{e}^{C T} \leqslant C K^{2}(\alpha, \gamma, \xi) .
$$

Thus, on $\left[0, T_{2}[\right.$,

$$
I^{2}(t) \leqslant C K^{2}(\alpha, \gamma, \xi)+\beta^{2}\left(C K^{2}(\alpha, \gamma, \xi)\right)+C \int_{0}^{t} I^{2}(s) \mathrm{d} s .
$$

Thanks to Gronwall's lemma,

$$
\sup _{\left[0, T_{2}[\right.} I^{2}(t) \leqslant\left(C K^{2}(\alpha, \gamma, \xi)+\beta^{2}\left(K^{2}(\alpha, \gamma, \xi)\right)\right) \mathrm{e}^{C T} .
$$

Iterating this argument, we deduce the existence of a function $\eta(u): \mathbb{R}^{+} \mapsto \mathbb{R}^{+}$, decreasing to 0 when $u$ decreases to 0 , such that

$$
\sup _{[0, T]} I(t) \leqslant \eta(K(\alpha, \gamma, \xi))
$$

Hence, there exists $\delta>0$ such that if $K(\alpha, \gamma, \xi) \leqslant \delta$, then $\sup _{[0, T]} I(t) \leqslant \varepsilon / 2$. It now suffices to choose $\alpha, \gamma, \xi$ small enough, such that

$$
K(\alpha, \gamma, \xi) \leqslant \delta, \quad 2 \alpha / \gamma \leqslant \varepsilon / 2,
$$

which will imply, for all $\omega \in \Omega(\alpha, \xi)$,

$$
\delta\left(X_{h}(\omega), S\right) \leqslant\|I(\omega)\|_{\infty}+\|\| \lambda(\omega) \| \mid \leqslant \varepsilon .
$$

First, we choose $\xi \in] 0, \xi\left(z_{1}\right) \wedge \ldots \wedge \xi\left(z_{n}\right)[$ small enough, in order to get $\varphi(\xi) \leqslant \delta / 3$. Then we choose $\gamma$ in $] 0,\left(\zeta_{0} / 8\right) \wedge(\delta / 3)^{6}[$. Finally, we choose

$$
0<\alpha<\gamma / 2 \wedge\left(\delta \gamma^{3 / 2} / 3\right)^{4} \wedge \varepsilon \gamma / 4
$$

Proposition 3.2 is proved. 


\section{The case where $N$ is 'deterministic'}

It remains to prove Proposition 3.3. In this entire section,

$$
m(\mathrm{~d} t, \mathrm{~d} z)=\sum_{i=1}^{n} \delta_{\left(t_{i}, z_{i}\right)}(\mathrm{d} t, \mathrm{~d} z) \in \mathscr{l l}
$$

is fixed. We set $t_{0}=0, t_{n+1}=T$.

We have to establish a support theorem for the solution of (3.3). Let us observe that this equation is not much different from that of Walsh (1986). Indeed, it only contains one additional term, a 'jump drift'. Nevertheless, it is far from possible to use a method similar to that of Bally et al. (1995), who proved a support theorem for Walsh's equation, in particular because the solution of (3.3) does not lie in $C([0, T] \times[0,1])$.

But the jump times of the solution $X_{m}$ of equation (3.3) are deterministic, and the associated skeleton $S(h, m)$ ( $m$ is fixed) has the same jump times. Thus we do not need the Skorokhod topology: we will work with the stronger supremum norm on $[0, T] \times[0,1]$.

The method below consists in applying the result of Bally et al. on each time interval $\left[t_{i}, t_{i+1}\left[\right.\right.$. To this end, we will define some processes $X_{m}^{i}$, which equal $X_{m}$ only on $\left[t_{i}, t_{i+1}\left[\times[0,1]\right.\right.$, but also give information about the behaviour of $X_{m}$ after $t_{i+1}$. We will also associate with $X_{m}^{i}$ some deterministic skeletons $S_{m}^{i}(h)$. But we will apply the result of Bally et al. (1995) to the conditional law of $X_{m}^{i}$ with respect to $\mathscr{F}_{t_{i}}$ (for each $i$ ). Thus, we will have to define a non-deterministic 'conditional skeleton' $T_{m}^{i}(h)$. Then we will develop a technical way to 'paste the pieces together'.

Recall that, thanks to Remark 2.4, we have to prove, on the one hand, that for all $h \in \mathscr{H}$, all $\varepsilon>0$,

$$
P\left(\left\|X_{m}-S(h, m)\right\|_{\infty} \leqslant \varepsilon\right)>0
$$

and, on the other hand, that

$$
P\left(X_{m} \in \overline{\{S(h, m) ; h \in \mathscr{H}\}}\left\|^{\|}\right\|_{\infty}\right)=1
$$

To this end, we introduce some notation. First, if $S(t, x)$ belongs to $\mathbb{D}([0, T], C([0,1]))$, and if $0 \leqslant u<v \leqslant T$, then

$$
\|S\|_{[u, v]}=\sup _{t \in[u, v], x \in[0,1]}|S(t, x)| .
$$

We now define recursively, for $i$ in $\{0, \ldots, n\}$, the processes $X_{m}^{i}(t, x)$ on $\left[t_{i}, T\right] \times[0,1]$,

$$
X_{m}^{0}(t, x)=G_{t}\left(\mathscr{C}_{0}, x\right)+\int_{0}^{t_{1} \wedge t} \int_{0}^{1} G_{t-s}(x, y)\left[b\left(X_{m}^{0}(s, y)\right) \mathrm{d} y \mathrm{~d} s+\sigma\left(X_{m}^{0}(s, y)\right) W(\mathrm{~d} y, \mathrm{~d} s)\right]
$$

and, for $i \in\{1, \ldots, n\}$, 


$$
\begin{aligned}
X_{m}^{i}(t, x)= & X_{m}^{i-1}(t, x)+\mathbf{1}_{\left\{t \geqslant t_{i}\right\}} \int_{0}^{1} G_{t-t_{i}}(x, y) g\left(X_{m}^{i-1}\left(t_{i}-, y\right), z_{i}\right) \mathrm{d} y \\
& +\int_{t_{i}}^{t_{i+1} \wedge t} \int_{0}^{1} G_{t-s}(x, y)\left[b\left(X_{m}^{i}(s, y)\right) \mathrm{d} y \mathrm{~d} s+\sigma\left(X_{m}^{i}(s, y)\right) W(\mathrm{~d} y, \mathrm{~d} s)\right] .
\end{aligned}
$$

Notice that, for all $i$,

$$
\text { for all } t \in\left[t_{i}, t_{i+1}\left[, \quad \text { all } x \in[0,1], \quad X_{m}^{i}(t, x)=X_{m}(t, x) .\right.\right.
$$

Indeed, it suffices to use a standard uniqueness argument. In the same way, we define, for $h \in \mathscr{H}$, the functions $S_{m}^{i}(h)$ on $\left[t_{i}, T\right] \times[0,1]$, by

$$
\begin{aligned}
& S_{m}^{0}(h)(t, x) \\
& \quad=G_{t}\left(\mathscr{C}_{0}, x\right)+\int_{0}^{t_{1} \wedge t} \int_{0}^{1} G_{t-s}(x, y)\left[b\left(S_{m}^{0}(h)(s, y)\right) \mathrm{d} y \mathrm{~d} s+\sigma\left(S_{m}^{0}(h)(s, y)\right) \dot{h}(s, y) \mathrm{d} y \mathrm{~d} s\right]
\end{aligned}
$$

and, for $i \in\{1, \ldots, n\}$,

$$
\begin{aligned}
S_{m}^{i}(h)(t, x)= & S_{m}^{i-1}(h)(t, x)+\mathbf{1}_{\left\{t \geqslant t_{i}\right\}} \int_{0}^{1} G_{t-t_{i}}(x, y) g\left(S_{m}^{i-1}(h)\left(t_{i}-, y\right), z_{i}\right) \mathrm{d} y \\
& +\int_{t_{i}}^{t_{i+1} \wedge t} \int_{0}^{1} G_{t-s}(x, y)\left[b\left(S_{m}^{i}(h)(s, y)\right) \mathrm{d} y \mathrm{~d} s+\sigma\left(S_{m}^{i}(h)(s, y)\right) \dot{h}(s, y) \mathrm{d} y \mathrm{~d} s\right] .
\end{aligned}
$$

Then, for all $i$,

$$
\text { for all } t \in\left[t_{i}, t_{i+1}\left[, \quad \text { all } x \in[0,1], \quad S_{m}^{i}(h)(t, x)=S(h, m)(t, x) .\right.\right.
$$

Finally, we define the 'conditional skeleton' associated with the conditional law of $X_{m}^{i}$ with respect to $\mathscr{F}_{t_{i}}$ :

$$
\begin{aligned}
T_{m}^{i}(h)(t, x)= & X_{m}^{i-1}(t, x)+\mathbf{1}_{\left\{t \geqslant t_{i}\right\}} \int_{0}^{1} G_{t-t_{i}}(x, y) g\left(X_{m}^{i-1}\left(t_{i}-, y\right), z_{i}\right) \mathrm{d} y \\
& +\int_{t_{i}}^{t_{i+1} \wedge t} \int_{0}^{1} G_{t-s}(x, y)\left[b\left(T_{m}^{i}(h)(s, y)\right) \mathrm{d} y \mathrm{~d} s+\sigma\left(T_{m}^{i}(h)(s, y)\right) \dot{h}(s, y) \mathrm{d} y \mathrm{~d} s\right] .
\end{aligned}
$$

The function $T_{m}^{i}(h)$ is defined on $\left[t_{i}, T\right] \times[0,1]$. For all $t\left[t_{i}, T\right]$, all $x \in[0,1], T_{m}^{i}(h)(t, x)$ is $\mathscr{F}_{t_{i}}$-measurable.

Then one can 'nearly' use the theorem of Bally et al. (1995) - for a more general setting, see Cardon-Weber and Millet (1999) - which yields the following result.

Proposition 5.1. Assume $(H)$ and $\left(S^{\prime} 1\right)$. Then, with the above notation, for all $i \in$ $\{0, \ldots, n\}$, the following conditional support theorem on $\left[t_{i}, T\right] \times[0,1]$ holds:

$$
\operatorname{supp}_{\|\|_{\left[t_{i}, T\right]}} \mathscr{C}\left(X_{m}^{i} \mid \mathscr{F}_{t_{i}}\right)=\overline{\left\{T_{m}^{i}(h) \mid h \in \mathscr{H}\right\}} \|_{\left[t_{i}, T\right]} .
$$


In fact, the main theorem in Bally et al. (1995) only yields the result for $i=0$, with $\int_{0}^{t}$ instead of $\int_{0}^{t \wedge t_{1}}$. But conditioning is not a problem, and the initial values we obtain, for example

$X_{m}^{i-1}(t, x)+\int_{0}^{1} G_{t-t_{i}}(x, y) g\left(X_{m}^{i-1}\left(t_{i}-, y\right), z_{i}\right) \mathrm{d} y=X_{m}^{i-1}(t, x)+G_{t-t_{i}}\left(g\left(X_{m}^{i-1}\left(t_{i}-,.\right), z_{i}\right), x\right)$,

behave on $\left[t_{i}, T\right]$ exactly as $G_{t}\left(\mathscr{C}_{0}, x\right)$ on $[0, T]$, since they are $\mathscr{F}_{t_{i}}$-measurable, since $g\left(X_{m}^{i-1}\left(t_{i}-,.\right), z_{i}\right)$ is continuous on $[0,1]$, and since $X_{m}^{i-1}(t, x)$ is continuous on $[0, T]$ $\times[0,1]$. Finally, it is clear that considering the integrals from $t_{i}$ to $t \wedge t_{i+1}$ instead of 0 to $t$ will not change much.

We now establish a lemma, which will allow us to paste the pieces together. If $\left\|X_{m}^{i}(\omega)-S_{m}^{i}(h)\right\|_{\left[t_{i}, T\right]}$ is small, then the initial positions associated with $S_{m}^{i+1}(h)$ and $T_{m}^{i+1}(h)(\omega)$ are near, and thus the distance between $S_{m}^{i+1}(h)$ and $T_{m}^{i+1}(h)(\omega)$ is small. We need this lemma because Proposition 5.1 gives an idea of the distance between $X_{m}^{i}(\omega)$ and $T_{m}^{i}(h)(\omega)$, but what we need to control is the distance between $S_{m}^{i}(h)$ and $X_{m}^{i}(\omega)$.

Lemma 5.2. Assume $(H)$ and $\left(S^{\prime} 2\right)$. There exists a function $\gamma(x, u): \mathbb{R}^{+} \times \mathbb{R}^{+} \mapsto \mathbb{R}^{+}$, such that, for each $x, \gamma(x, u)$ decreases to 0 when $u$ decreases to 0 , and such that, for all $\varepsilon>0$, all $i \in\{0, \ldots, n-1\}$,

$$
\begin{aligned}
& \left\{\omega \in \Omega ;\left\|X_{m}^{i}(\omega)-S_{m}^{i}(h)\right\|_{\left[t_{i}, T\right]} \leqslant \varepsilon\right\} \\
& \quad \subset\left\{\omega \in \Omega ;\left\|S_{m}^{i+1}(h)-T_{m}^{i+1}(h)(\omega)\right\|_{\left[t_{i+1}, T\right]} \leqslant \gamma\left(\left\|\left.\dot{h}\right|_{\left[t_{i+1}, t_{i+2}\right]}\right\|_{L^{2}}, \varepsilon\right)\right\}
\end{aligned}
$$

where $\left\|\left.\dot{h}\right|_{\left[t_{i+1}, t_{i+2}\right]}\right\|_{L^{2}}^{2} \int_{t_{i+1}}^{t_{i+2}}=\int_{0}^{1} \dot{h}^{2}(s, y) \mathrm{d} y \mathrm{~d} s$.

Proof. Let $\omega$ belong to $\left\{\left\|X_{m}^{i}-S_{m}^{i}(h)\right\|_{\left[t_{i}, T\right]} \leqslant \epsilon\right\}$. Then, for all $t$ in $\left[t_{i+1}, T\right]$, all $x$ in $[0,1]$, using $(H)$,

$$
\begin{aligned}
& \left|S_{m}^{i+1}(h)(t, x)-T_{m}^{i+1}(h)(t, x)\right| \leqslant\left|S_{m}^{i}(h)(t, x)-X_{m}^{i}(t, x)\right| \\
& \quad+\int_{0}^{1} G_{t-t_{i+1}}(x, y)\left|g\left(X_{m}^{i}\left(t_{i+1}-, y\right), z_{i}\right)-g\left(S_{m}^{i}(h)\left(t_{i+1}-, y\right), z_{i}\right)\right| \mathrm{d} y \\
& \quad+C \int_{t_{i+1}}^{t_{i+2} \wedge t} \int_{0}^{1} G_{t-s}(x, y)\left|S_{m}^{i+1}(h)(s, y)-T_{m}^{i+1}(h)(s, y)\right|(1+|\dot{h}(s, y)|) \mathrm{d} y \mathrm{~d} s .
\end{aligned}
$$

We now set

$$
F(t)=\sup _{x \in[0,1]}\left|S_{m}^{i+1}(h)(t, x)-T_{m}^{i+1}(h)(t, x)\right| .
$$

Using the assumption about $\omega$, Assumption ( $\left.S^{\prime} 2\right)$, (A.2) and (A.1), and the Cauchy-Schwarz inequality, we obtain

$$
F(t) \leqslant \varepsilon+\psi_{z_{i}}(\varepsilon)+C\left(1+\left\|\left.\dot{h}\right|_{\left[t_{i+1}, t_{i+2}\right]}\right\|_{L^{2}}\right)\left(\int_{t_{i+1}}^{t} F^{2}(s) \frac{\mathrm{d} s}{\sqrt{t-s}}\right)^{1 / 2}
$$


where $\psi_{z_{i}}$ was defined in Assumption $\left(S^{\prime} 2\right)$. Hence,

$$
F^{2}(t) \leqslant C \varepsilon^{2}+C \psi_{z_{i}}^{2}(\varepsilon)+C\left(1+\left\|\left.\dot{h}\right|_{\left[t_{i+1}, t_{i+2}\right]}\right\|_{L^{2}}\right)^{2} \int_{t_{i+1}}^{t} F^{2}(s) \frac{\mathrm{d} s}{\sqrt{t-s}} .
$$

Iterating this formula once (see the previous section, inequalities (4.30), (4.31) and (4.32), for more details), we obtain the existence of a function $\gamma$, satisfying the assumptions of the statement, such that

$$
F^{2}(t) \leqslant \gamma\left(\left\|\left.\dot{h}\right|_{\left[t_{i+1}, t_{i+2}\right]}\right\|_{L^{2}}, \varepsilon\right)+C\left(1+\left\|\left.\dot{h}\right|_{\left[t_{i+1}, t_{i+2}\right]}\right\|_{L^{2}}\right)^{2} \int_{t_{i+1}}^{t} F^{2}(s) \mathrm{d} s .
$$

Gronwall's lemma allows us to conclude the proof.

In order to simplify notation, we assume in the following that $n=2$, i.e. that

$$
m(\mathrm{~d} t, \mathrm{~d} z)=\delta_{\left(t_{1}, z_{1}\right)}+\delta_{\left(t_{2}, z_{2}\right)} .
$$

We first fix $h \in \mathscr{H}$, and $\varepsilon>0$, and we check that

$$
P_{0}=P\left(\left\|X_{m}-S(h, m)\right\|_{\infty} \leqslant \varepsilon\right)>0 .
$$

Using (5.7) and (5.10), we see that

$$
P_{0} \geqslant P\left(\left\|X_{m}^{0}-S_{m}^{0}(h)\right\|_{[0, T]} \leqslant \varepsilon / 3,\left\|X_{m}^{1}-S_{m}^{1}(h)\right\|_{\left[t_{1}, T\right]} \leqslant \varepsilon / 3,\left\|X_{m}^{2}-S_{m}^{2}(h)\right\|_{\left[t_{2}, T\right]} \leqslant \varepsilon / 3\right) .
$$

Noticing that, for each $i, X_{m}^{i}$ is $\mathscr{F}_{t_{i+1}}$-measurable and $S_{m}^{i}(h)$ is deterministic, we obtain, by conditioning our probability with respect to $\mathscr{F}_{t_{2}}$,

$$
P_{0} \geqslant \mathrm{E}\left[\mathbf{1}_{\left\{\left\|X_{m}^{0}-S_{m}^{0}(h)\right\|_{[0, T]} \leqslant \varepsilon / 3\right\}} \rrbracket_{\left\{\left\|X_{m}^{1}-S_{m}^{1}(h)\right\|_{\left[t_{1}, T\right]} \leqslant \varepsilon / 3\right\}} P\left(\left\|X_{m}^{2}-S_{m}^{2}(h)\right\|_{\left[t_{2}, T\right]} \leqslant \varepsilon / 3 \mid \mathscr{F}_{t_{2}}\right)\right] .
$$

On the other hand,

$$
\begin{aligned}
& P\left(\left\|X_{m}^{2}-S_{m}^{2}(h)\right\|_{\left[t_{2}, T\right]} \leqslant \varepsilon / 3 \mid \mathscr{F}_{t_{2}}\right) \\
& \quad \geqslant P\left(\left\|X_{m}^{2}-T_{m}^{2}(h)\right\|_{\left[t_{2}, T\right]} \leqslant \varepsilon / 6,\left\|T_{m}^{2}(h)-S_{m}^{2}(h)\right\|_{\left[t_{2}, T\right]} \leqslant \varepsilon / 6 \mid \mathscr{F}_{t_{2}}\right) \\
& \quad \geqslant \mathbf{1}_{\left\{\left\|T_{m}^{2}(h)-S_{m}^{2}(h)\right\|_{\left[t_{2}, T\right]} \leqslant \epsilon\right\}} P\left(\left\|X_{m}^{2}-T_{m}^{2}(h)\right\|_{\left[t_{2}, T\right]} \leqslant \epsilon / 6 \mid \mathscr{F}_{t_{2}}\right),
\end{aligned}
$$

since $S_{m}^{2}(h)$ is deterministic and $T_{m}^{2}(h)$ is $\mathscr{F}_{t_{2}}$-measurable. Using Proposition 5.1, we also know that

$$
P\left(\left\|X_{m}^{2}-T_{m}^{2}(h)\right\|_{\left[t_{2}, T\right]} \leqslant \epsilon / 6 \mid \mathscr{F}_{t_{2}}\right)>0
$$

almost surely. Hence, it suffices that $P_{1}>0$, where

$$
P_{1}=P\left(\left\|X_{m}^{0}-S_{m}^{0}(h)\right\|_{[0, T]} \leqslant \epsilon / 3,\left\|X_{m}^{1}-S_{m}^{1}(h)\right\|_{\left[t_{1}, T\right]} \leqslant \varepsilon / 3,\left\|T_{m}^{2}(h)-S_{m}^{2}(h)\right\|_{\left[t_{2}, T\right]} \leqslant \varepsilon / 6\right) .
$$


Thanks to Lemma 5.2, we know that, for $\alpha>0$ small enough,

$$
\left\|X_{m}^{1}-S_{m}^{1}(h)\right\|_{\left[t_{1}, T\right]}<\alpha \Rightarrow\left\|T_{m}^{2}(h)-S_{m}^{2}(h)\right\|_{\left[t_{2}, T\right]} \leqslant \varepsilon / 6 .
$$

Thus,

$$
P_{1} \geqslant P\left(\left\|X_{m}^{0}-S_{m}^{0}(h)\right\|_{[0, T]} \leqslant \epsilon / 3,\left\|X_{m}^{1}-S_{m}^{1}(h)\right\|_{\left[t_{1}, T\right]} \leqslant \alpha \wedge \varepsilon / 3\right) .
$$

Iterating this argument, we see that $P_{0}$ is strictly positive as soon as $P_{2}>0$, where

$$
P_{2}=P\left(\left\|X_{m}^{0}-S_{m}^{0}(h)\right\|_{[0, T]} \leqslant \beta\right)
$$

for some $\beta>0$ small enough. But it is clear that $S_{m}^{0}(h)$ is identically equal to $T_{m}^{0}(h)$. Thus, Proposition 5.1 implies that $P_{2}$ is strictly positive, and hence that (5.3) holds, which was our aim.

We still have to check that

$$
P\left(X_{m} \in \overline{\{S(h, m), h \in \mathscr{H}\}^{\|} \|_{\infty}}\right)=1 .
$$

We know from Proposition 5.1 that, for almost all $\omega$, say for all $\omega \in \tilde{\Omega}$, with $P(\bar{\Omega})=1$,

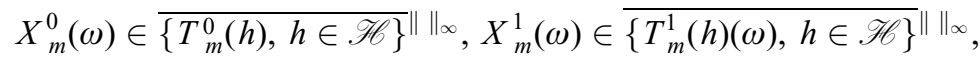

$$
\begin{aligned}
& X_{m}^{2}(\omega) \in \overline{\left\{T_{m}^{2}(h)(\omega), h \in \mathscr{H}\right\}} \|^{\|_{\infty}} .
\end{aligned}
$$

We now fix $\omega \in \bar{\Omega}$. There exists $h_{n}^{0} \in \mathscr{H}, h_{n}^{1} \in \mathscr{H}, h_{n}^{2} \in \mathscr{H}$ (depending on $\omega$ ), such that, for $i \in\{0,1,2\}$, when $n$ goes to infinity,

$$
\left\|X_{m}^{i}(\omega)-T_{m}^{i}\left(h_{n}^{i}\right)(\omega)\right\|_{\left[t_{i}, T\right]} \rightarrow 0 .
$$

We now set

$$
h_{n, k, q}(t, x)=h_{n}^{0}(t, x) \mathbf{1}_{\left[0, t_{1}\right]}(t)+h_{k}^{1}(t, x) \mathbf{1}_{\left[t_{1}, t_{2}\right]}(t)+h_{q}^{2}(t, x) \mathbf{1}_{\left[t_{2}, T\right]}(t) .
$$

We fix $\varepsilon>0$, and we prove that, for $n, k, q$ large enough,

$$
\left\|X_{m}(\omega)-S\left(h_{n, k, q}, m\right)\right\|_{[0, T]} \leqslant \varepsilon,
$$

which will suffice. One can easily check, using (5.1) and (5.2), that

$$
\left\|X_{m}(\omega)-S\left(h_{n, k, q}, m\right)\right\|_{[0, T]} \leqslant A_{n}^{0}(\omega)+A_{k}^{1}(\omega)+A_{q}^{2}(\omega)+B_{n}^{0}(\omega)+B_{k}^{1}(\omega)+B_{q}^{2}(\omega),
$$

where (if $i=0,1,2$ and $l \in \mathbb{N}$ )

$$
A_{l}^{i}(\omega)=\left\|X_{m}^{i}(\omega)-T_{m}^{i}\left(h_{l}^{i}\right)(\omega)\right\|_{\left[t_{i}, T\right]}
$$

and

$$
B_{l}^{i}(\omega)=\left\|T_{m}^{i}\left(h_{l}^{i}\right)(\omega)-S_{m}^{i}\left(h_{l}^{i}\right)\right\|_{\left[t_{i}, T\right]} .
$$

Notice that $B_{n}^{0}$ vanishes identically. Thanks to Lemma 5.2, we know that

$$
B_{k}^{1}(\omega) \leqslant \gamma\left(\left\|\left.\dot{h}_{k}^{1}\right|_{\left[t_{1}, t_{2}\right]}\right\|_{L^{2}}, A_{n}^{0}(\omega)\right),
$$




$$
B_{q}^{2}(\omega) \leqslant \gamma\left(\left\|\left.\dot{h}_{q}^{2}\right|_{\left[t_{2}, T\right]}\right\|_{L^{2}}, A_{k}^{1}(\omega)+B_{k}^{1}(\omega)\right) .
$$

First, we choose $q$ large enough, in order that

$$
A_{q}^{2}(\omega) \leqslant \varepsilon / 6 .
$$

Now that $q$ is fixed, we consider $\alpha>0$ such that

$$
\gamma\left(\left\|\left.\dot{h}_{q}^{2}\right|_{\left[t_{2}, T\right]}\right\|_{L^{2}}, \alpha\right) \leqslant \varepsilon / 6 .
$$

Then we choose $k$ in such a way that

$$
A_{k}^{1}(\omega) \leqslant \varepsilon / 6 \wedge \alpha / 2
$$

and we consider $\beta>0$ such that

$$
\gamma\left(\left\|\left.\dot{h}_{k}^{1}\right|_{\left[t_{1}, t_{2}\right]}\right\|_{L^{2}}, \beta\right) \leqslant \varepsilon / 6 \wedge \alpha / 2 .
$$

Finally, we choose $n$ such that

$$
A_{n}^{0}(\omega) \leqslant \varepsilon / 6 \wedge \beta
$$

We deduce from (5.13), (5.7) and (5.12) that

$$
B_{k}^{1}(\omega) \leqslant \varepsilon / 6 \wedge \alpha / 2 .
$$

Thanks to (5.14), (5.11), (5.10) and (5.8), we also see that

$$
B_{q}^{2}(\omega) \leqslant \varepsilon / 6
$$

Finally, using (5.6), (5.13), (5.11), (5.9), (5.14) and (5.15), we deduce (5.5). We have thus checked that for each $\omega \in \tilde{\Omega}$, all $\varepsilon>0$, there exists $h \in \mathscr{H}$ such that

$$
\left\|X_{m}(\omega)-S(h, m)\right\|_{\infty} \leqslant \varepsilon .
$$

Since $P(\tilde{\Omega})=1,(5.4)$ holds and Proposition 3.3 is proved.

\section{Extension to the case of an almost surely infinite number of jumps when the diffusion coefficient is constant}

We now consider equation (1.1) in the following new setting: the diffusion coefficient is constant, $\sigma(x) \equiv \sigma$; but the positive measure $q$ on $E$ is only assumed to be $\sigma$-finite (a priori, $q(E)=\infty) . N$ is still a Poisson measure on $[0, T] \times E$, with intensity measure $\mathrm{d} t q(\mathrm{~d} z)$. The evolution equation associated with equation (1.1) is still given by (2.4).

We also consider an increasing sequence of subsets $E_{p}$ of $E$ satisfying

$$
q\left(E_{p}\right)<\infty, \quad \cup_{p \in \mathbb{N}} E_{p}=E .
$$

In order to obtain a result of existence and uniqueness, we state the following hypothesis:

Assumption (A). The function $\sigma$ is constant. The function $b$ satisfies a global Lipschitz condition. There exists $\eta \in L^{1}(E, q)$ such that for all $x, y \in \mathbb{R}$, all $z \in E$, 


$$
|g(0, z)| \leqslant \eta(z), \quad|g(x, z)-g(y, z)| \leqslant|x-y| \eta(z)
$$

Proposition 2.2 yields that equation (1.1) with $E_{p}$ instead of $E$ admits a unique weak solution $X^{p}$ lying in $\mathbb{D}([0, T], C([0,1]))$. Under Assumption $(A)$, using strongly the fact that $\sigma$ is constant, it is easy to check that there exists an adapted process $X$ such that, when $p$ goes to infinity,

$$
\mathrm{E}\left(\sup _{[0, T] \times[0,1]}\left|X(t, x)-X^{p}(t, x)\right|\right) \rightarrow 0 .
$$

In this way, we obtain the existence of an adapted weak solution $X$ of (1.1) with our new setting. See Remark 6.6 for the case where $\sigma$ is not a constant.

The uniqueness is straightforward under $(A)$, and we can state the following proposition.

Proposition 6.1. Assume (A). Equation (1.1) admits a unique weak solution $X(t, x)$, lying almost surely in $\mathbb{D}([0, T], C([0,1]))$, and bounded in $L^{1}$.

We now consider

$$
\begin{aligned}
& \mathscr{C l}_{p}= \\
& \left.\qquad m(\mathrm{~d} t, \mathrm{~d} z)=\sum_{i=1}^{n} \delta_{\left(t_{i}, z_{i}\right)}(\mathrm{d} t, \mathrm{~d} z) \mid n \in \mathbb{N}, 0<t_{1}<\ldots<t_{n}<T, z_{1}, \ldots, z_{n} \in \operatorname{supp} q \cap E_{p}\right\}
\end{aligned}
$$

and we set $\mathscr{C}=\cup_{p} \mathscr{C l}_{p}$. The Cameron-Martin space $\mathscr{H}$ associated with $W$ is still defined by (2.5). For each $m \in \mathscr{L}$ and $h \in \mathscr{H}$, we denote by $S(h, m)$ the unique solution of equation (2.6) (there is no difference between the situation here and in Proposition 2.3, since there exists $p$ such that $m \in \mathscr{C}_{p}$ ). Since $g$ is already Lipschitz, we assume $(T)$ below instead of $(S 2)$ :

Assumption (T). For each $z_{0} \in E$, each $n \in \mathbb{N}$,

$$
\sup _{|x| \leqslant n}\left|g(x, z)-g\left(x, z_{0}\right)\right| \rightarrow d\left(z, z_{0}\right) \rightarrow 00 .
$$

For each $z_{0}$ in $E$, there exists $\xi\left(z_{0}\right)>0$ such that

$$
\sup _{d\left(z, z_{0}\right) \leqslant \xi\left(z_{0}\right)} \eta(z)<\infty .
$$

A function $g(x, z)=\alpha(z) \eta(z)$ clearly satisfies $(A)$ and $(T)$ if $\alpha$ is Lipschitz, and $\eta \in$ $L^{1}(E, q)$ is continuous. The aim of this section is to prove the following result.

Theorem 6.2. Under $(A)$ and $(T)$, if $X$ denotes the unique weak solution of equation (1.1),

$$
\operatorname{supp}_{\delta} P \circ X^{-1}=\overline{\{S(h, m) \mid h \in \mathscr{H}, m \in \mathscr{C}\}^{\delta}} .
$$


Since the method of Simon (1999), combined with the previous sections, can be easily applied, we will only sketch the proof.

First, for the same reasons as in the previous sections (see Proposition 3.1), we can assume, in addition to $(A)$ and $(T)$, that for all $x \in \mathbb{R}$, all $z \in E,|g(x, z)| \leqslant \eta(z)$, and that for each $z_{0} \in E$,

$$
\sup _{x \in \mathbb{R}}\left|g(x, z)-g\left(x, z_{0}\right)\right| \rightarrow_{d\left(z, z_{0}\right) \rightarrow 0} 0 .
$$

Then, we notice that the direct inclusion $(\subset)$ of Theorem 6.2 is immediate, thanks to Theorem 2.5 (for $X^{p}$ ) and thanks to the convergence (6.1).

We now fix $p \in \mathbb{N}, h \in \mathscr{H}, m=\sum_{i=1}^{n} \delta_{\left(t_{i}, z_{i}\right)} \in \mathscr{L}_{p}$ and $\varepsilon>0$. We have to prove that

$$
P(\delta(X, S(h, m)) \leqslant \varepsilon)>0 .
$$

To do so, we will use three lemmas. The first one is a very particular case of the result of Bally et al. (1995).

Lemma 6.3. Let $\alpha>0$ be fixed, and let

$$
\Omega_{0}(\alpha)=\left\{\omega \in \Omega ; \sup _{t, x}\left|\int_{0}^{t} \int_{0}^{1} G_{t-s}(x, y)\{W(\mathrm{~d} y, \mathrm{~d} s)-\dot{h}(s, y) \mathrm{d} y \mathrm{~d} s\}\right| \leqslant \alpha\right\} .
$$

Then $P\left(\Omega_{0}(\alpha)\right)>0$.

We now write the restriction $N^{p}=\left.N\right|_{[0, T] \times E p}$ (recall that $p$ is fixed) as

$$
N^{p}(\mathrm{~d} s, \mathrm{~d} z)=\sum_{i=1}^{\mu} \delta_{\left(T_{i}, Z_{i}\right)}(\mathrm{d} s, \mathrm{~d} z) .
$$

The second lemma can be proved by using the same method as that of Proposition 3.2 (see Section 4). The only difference comes from the fact that $X^{p}(\omega)$ depends on $W$, but since $\sigma$ is constant, Lemma 6.3 allows us to deal with this problem easily.

Lemma 6.4. Let $\beta>0$ be fixed. There exists a set

$$
\Omega_{1}(\beta) \in \sigma\left\{N(A) ; A \in \mathscr{B}\left([0, T] \times E_{p}\right)\right\}
$$

such that $P\left(\Omega_{1}(\beta)\right)>0$, such that for each $\omega \in \Omega_{1}(\beta)$,

$$
\mu(\omega)=n, \quad \forall i, \quad d\left(z_{i}, Z_{i}(\omega)\right) \leqslant \xi\left(z_{i}\right),
$$

and such that for some $\alpha>0$ small enough, every $\omega \in \Omega_{0}(\alpha) \cap \Omega_{1}(\beta)$ satisfies

$$
\delta\left(X^{p}(\omega), S(h, m)\right) \leqslant \beta .
$$

The third lemma we require, along with its proof, is as follows.

Lemma 6.5. Let $\gamma>0$ be fixed, and let 


$$
\Omega_{2}(\gamma)=\left\{\omega \in \Omega ; \int_{0}^{T} \int_{E / E_{p}} \eta(z) N(\mathrm{~d} s, \mathrm{~d} z) \leqslant \gamma\right\} .
$$

Then $P\left(\Omega_{2}(\gamma)\right)>0$.

Proof. We set

$$
\theta_{p}=\int_{0}^{T} \int_{E / E_{p}} \eta(z) N(\mathrm{~d} s, \mathrm{~d} z)
$$

and, for $q>p$,

$$
\theta_{p}^{q}=\int_{0}^{T} \int_{E_{q} / E_{p}} \eta(z) N(\mathrm{~d} s, \mathrm{~d} z) .
$$

We see that $\theta_{p}=\theta_{p}^{q}+\theta_{q}$, that for all $q, P\left(\theta_{p}^{q}=0\right)>0$, and that when $q$ goes to infinity, $\theta_{q}$ goes to 0 in probability. Since, for each $q, \theta_{p}^{q}$ is independent of $\theta_{q}$, we can write

$$
P\left(\theta_{p} \leqslant \gamma\right) \geqslant P\left(\theta_{p}^{q}=0\right) P\left(\theta_{q} \leqslant \gamma\right)
$$

and the lemma follows easily.

We finally sketch the proof of Theorem 6.2. An easy independence argument yields that for every $\alpha>0, \beta>0, \gamma>0$, the set

$$
\Omega_{3}(\alpha, \beta, \gamma)=\Omega_{0}(\alpha) \cap \Omega_{1}(\beta) \cap \Omega_{2}(\gamma)
$$

has strictly positive probability. We now have to to choose $\alpha, \beta, \gamma$ in such a way that, for all $\omega \in \Omega_{3}(\alpha, \beta, \gamma)$,

$$
\delta(X(\omega), S(h, m)) \leqslant \varepsilon .
$$

Let $\omega \in \Omega_{3}(\alpha, \beta, \gamma)$ be fixed. If $\alpha$ is small enough, we know from Lemma 6.4 that

$$
\begin{aligned}
\delta(X(\omega), S(h, m)) & \leqslant\left\|X(\omega)-X^{p}(\omega)\right\|_{\infty}+\delta\left(X^{p}(\omega), S(h, m)\right) \\
& \leqslant\left\|X(\omega)-X^{p}(\omega)\right\|_{\infty}+\beta .
\end{aligned}
$$

We now set

$$
V^{p}(t)=\sup _{x \in[0,1]}\left|X(t, x)-X^{p}(t, x)\right| .
$$

Using the Appendix and Assumptions $(A)$ and $(T)$, since $|g(x, z)| \leqslant \eta(z)$, and since $\omega$ belongs to $\Omega_{3}(\alpha, \beta, \gamma)$, we see that

$$
\begin{aligned}
V^{p}(t) & \leqslant C \int_{0}^{t} V^{p}(s) \mathrm{d} s+C \sum_{i=1}^{n} 1_{\left\{t \geqslant T_{i}\right\}} V^{p}\left(T_{i}-\right) \eta\left(Z_{i}\right)+\int_{0}^{T} \int_{E \backslash E_{p}} \eta(z) N(\mathrm{~d} s, \mathrm{~d} z) \\
& \leqslant C \int_{0}^{t} V^{p}(s) \mathrm{d} s+C \sum_{i=1}^{n} 1_{\left\{t \geqslant T_{i}\right\}} V^{p}\left(T_{i}-\right)+\gamma .
\end{aligned}
$$


For the second term, we have used (6.2) in Assumption (T), and the fact that for all $i, Z_{i}$ belongs to $\left\{z \in E, d\left(z_{i}, z\right) \leqslant \xi\left(z_{i}\right)\right\}$.

Applying Gronwall's lemma successively on the time intervals $\left[0, T_{1}\left[, \ldots,\left[T_{n}, T\right]\right.\right.$, we deduce that, for all $\omega \in \Omega_{3}(\alpha, \beta, \gamma)$,

$$
\sup _{[0, T]} V^{p}(\omega, t) \leqslant C \gamma .
$$

The conclusion follows easily.

Remark 6.6. Of course, we are also interested in the case where $q(E)=\infty$ and $\sigma$ is a function. In this case, it is possible to prove (under certain assumptions) that the sequence $X_{p}$ of weak solutions of (1.1) where we have replaced $E$ by $E_{p}$, converges to an adapted process $X(t, x)$ in the following sense:

$$
\sup _{t, x} \mathrm{E}\left(\left|X(t, x)-X^{p}(t, x)\right|\right) \rightarrow 0 .
$$

Once $X$ is constructed, it might be possible to check that it admits a modification lying in $\mathbb{D}([0, T], C([0,1]))$, by using the fact that $X$ satisfies the evolution equation, but this is not immediate. If so, it seems natural to think that our support theorem extends to this case. However, everything will become much more technical. In particular, the direct inclusion is no longer obvious, since (6.1) no longer seems to hold.

\section{Appendix}

We collect here well-known estimates for the Green kernel $G_{t}(x, y)$ associated with the deterministic system (2.1), and given by (2.2). In all the inequalities below, the constant $C$ depends only on the terminal time value $T$. The first three estimates can be found in Walsh (1986) and the last three are either easy consequences or classical estimates.

First, for all $x, y \in[0,1]$ and all $t \in[0, T]$,

$$
\frac{1}{\sqrt{4 \pi t}} \exp \left\{\frac{-(y-x)^{2}}{4 t}\right\} \leqslant G_{t}(x, y) \leqslant \frac{C}{\sqrt{t}} \exp \left\{\frac{-(y-x)^{2}}{4 t}\right\} .
$$

For all $0<t<T$, all $x \in[0,1]$,

$$
\int_{0}^{1} G_{t}^{2}(x, y) \mathrm{d} y \leqslant \frac{C}{\sqrt{t}}
$$

and

$$
\int_{0}^{1} G_{t}(x, y) \mathrm{d} y=1 .
$$

For all $0<s<t<T$, all $x, y \in[0,1]$ (see Chenal and Millet 1997, Lemma A3)

$$
\left|G_{t}(x, y)-G_{s}(x, y)\right| \leqslant C \frac{|t-s|}{s^{3 / 2}}
$$


and (see Bally et al. 1995, Lemma B1)

$$
\int_{0}^{s} \int_{0}^{1}\left(G_{t-r}(x, y)-G_{s-r}(x, y)\right)^{2} \mathrm{~d} y \mathrm{~d} r+\int_{s}^{t} \int_{0}^{1} G_{t-r}^{2}(x, y) \mathrm{d} y \mathrm{~d} r \leqslant C \sqrt{t-s} .
$$

Finally, for all $\phi \in C([0,1])$, the map

$$
(t, x) \mapsto G_{t}(\phi, x)
$$

is continuous on $[0, T] \times[0,1]$ (for a similar result, see Bally et al. 1995, Lemma A2).

\section{References}

Albeverio, S., Wu, J.L. and Zhang, T.S. (1998) Parabolic SPDEs driven by Poisson white noise. Stochastic Process. Appl., 74, 21-36.

Bally, V., Millet, A. and Sanz-Solé, M. (1995) Approximation and support theorem in Hölder norm for parabolic SPDEs. Ann. Probab., 23, 178-222.

Cardon-Weber, C. and Millet, A. (1999) A support theorem for a generalized Burgers SPDE. Prépublication 503, Laboratoire de Probabilités de Paris 6.

Chenal, F. and Millet, A. (1997) Uniform large deviations for parabolic SPDEs and applications. Stochastic Process. Appl., 72, 161-186.

Fournier, N. (2000) Malliavin Calculus for parabolic SPDEs with jumps. Stochastic Process. Appl., 87, $115-147$.

Ikeda, N. and Watanabe, S. (1979) Stochastic Differential Equations and Diffusion Processes. Amsterdam: North Holland.

Jacod, J. and Shiryaev, A.N. (1987) Limit Theorems for Stochastic Processes. Berlin: Springer-Verlag. Millet, A. and Sanz-Solé, M. (1994) A simple proof of the support theorem for diffusion processes. In J. Azéma, P.-A. Meyer and M. Yor (eds), Séminaire de Probabilités XXVIII, Lecture Notes in Math. 1583. pp. 36-48. Berlin: Springer-Verlag.

Saint Loubert Bié, E. (1998) Étude d'une EDPS conduite par un bruit Poissonnien. Thèse, Université de Clermont-Ferrand.

Simon, T. (1999) Support theorem for jump processes. Preprint, Université d'Evry.

Stroock, D.W. and Varadhan, S.R.S. (1972) On the support of diffusion processes with application to the strong maximum principle. In L. LeCam, J. Neyman and E.L. Scott (eds), Proceedings of the Sixth Berkeley Symposium on Mathematical Statistics and Probability, Vol. III. Berkeley: University of California Press.

Walsh, J.B. (1981) A stochastic model for neural response. Adv. Appl. Probab., 13, 231-281.

Walsh, J.B. (1986) An introduction to stochastic partial differential equations. In P.L. Hennequin (ed.), École d'Éte de Probabilités de Saint Flour XIV, Lecture Notes in Math. 1180, pp. 265-439. Berlin: Springer-Verlag.

Received September 1999 and revised April 2000 